Article

\title{
Buoyant Unstable Behavior of Initially Spherical Lean Hydrogen-Air Premixed Flames
}

\section{Zuo-Yu Sun, Guo-Xiu Li *, Hong-Meng Li, Yue Zhai and Zi-Hang Zhou}

School of Mechanical, Electronic and Control Engineering, Beijing Jiaotong University,

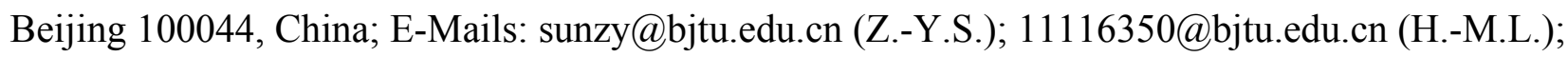
zhaiyue0000@163.com (Y.Z.); zhou_zihang@yahoo.com (Z.-H.Z.)

* Author to whom correspondence should be addressed; E-Mail: li_guoxiu@yahoo.com; Tel./Fax: +86-10-5168-4684.

Received: 31 March 2014; in revised form: 16 July 2014 / Accepted: 23 July 2014 /

Published: 31 July 2014

\begin{abstract}
Buoyant unstable behavior in initially spherical lean hydrogen-air premixed flames within a center-ignited combustion vessel have been studied experimentally under a wide range of pressures (including reduced, normal, and elevated pressures). The experimental observations show that the flame front of lean hydrogen-air premixed flames will not give rise to the phenomenon of cellular instability when the equivalence ratio has been reduced to a certain value, which is totally different from the traditional understanding of the instability characteristics of lean hydrogen premixed flames. Accompanied by the smoothened flame front, the propagation mode of lean hydrogen premixed flames transitions from initially spherical outwardly towards upwardly when the flames expand to certain sizes. To quantitatively investigate such buoyant instability behaviors, two parameters, "float rate $(\psi)$ " and "critical flame radius $\left(R_{\mathrm{cr}}\right)$ ", have been proposed in the present article. The quantitative results demonstrate that the influences of initial pressure $\left(P_{\text {int }}\right)$ on buoyant unstable behaviors are different. Based on the effects of variation of density difference and stretch rate on the flame front, the mechanism of such buoyant unstable behaviors has been explained by the competition between the stretch force and the results of gravity and buoyancy, and lean hydrogen premixed flames will display buoyant unstable behavior when the stretch effects on the flame front are weaker than the effects of gravity and buoyancy.
\end{abstract}


Keywords: lean hydrogen flame; initial spherical flame; buoyant behaviors; experimental observation

\section{Introduction}

With the concerns upon the depletion of fossil fuels and the deterioration of the global environment, investigations on alternative fuels have become a hot topic in the energy science field. Due to its preeminent advantages in burning velocity and emitted products, hydrogen gas has been considered as a promising alternative fuel for the future [1,2] and has been employed in spark-ignition (SI) internal combustion engines throughout the world [3-5]. For a SI internal combustion engine, the combustion performance is strongly affected by the propagation process, and hence an in-depth understanding of the characteristics of propagating hydrogen-air premixed flames is essential for designing and optimizing hydrogen fuelled SI engines [6,7], and it is widely believed that the intrinsic instabilities are of the utmost importance for premixed flames [8,9].

Over the past decades, the knowledge about the intrinsic instabilities of hydrogen premixed flames has been remarkably enriched by previous scholars' efforts. According to the previously reported results [10-13], the flame front of premixed hydrogen-air flames will be continuously wrinkled towards cellular instability during the propagation. There exist three intrinsic factors to induce an unstable premixed flame-hydrodynamic effects, thermal-diffusive effects, and buoyant effects [14]. The hydrodynamic instability is induced by the thermal expansion across the flame front [15], which has been proved to be present for all premixed flames and whose intensity is always indicated by the density ratio between the two sides of the flame front [8-14]; the thermal-diffusive instability is induced by the competing effects between heat conduction from the flame and the reactant diffusion towards the flame, and the intensity is always indicated by the Lewis number (which is defined as the ratio of the thermal diffusivity to the mass diffusivity) [16]; and the buoyant instability is commonly regarded as be relevant to body-force.

According to the results obtained by the previous scholars, for lean hydrogen-air premixed flames, with the decline of fuel concentration (usually indicated by the equivalence ratio $\varphi$, defined as the ratio of actual fuel-air ratio to stoichiometric fuel-air ratio), the density ratio across the flame front is reduced but the Lewis number is increased [8], which means the hydrodynamic instability will be weakened and the thermal-diffusive instability will be enhanced. As can be seen in Figure 1 (flame images during the propagation obtained in the present investigation), for the lean hydrogen premixed flames whose $\varphi$ falls into the range of 0.410 to 0.997 , with the decrease of $\varphi$, the flame front with a similar size is more wrinkled and the cellular behavior is more obvious. The enhancement of unstable behavior is the result of the enhanced thermal-diffusive instability rather than a declined hydrodynamic instability, and such a conclusion has been widely recognized by others [17-24]. However, from the comparison between the lean hydrogen premixed flames with $\varphi=0.265$ and $\varphi=0.410$, a distinct phenomenon can be observed whereby the flame front with a leaner fuel concentration is relatively smoother, which means the further enhanced thermal-diffusive instability has not made the leaner hydrogen premixed flame more cellular unstable. Furthermore, for the lean hydrogen premixed flame 
with $\varphi=0.265$, the initial spherical outward propagation will transit towards an upward displacement where the geometrical center of the flame is lifting up when it has propagated to a certain size, namely, the hydrogen premixed flame has become buoyant unstable. Although some scholars have investigated buoyant unstable flames by numerical methods [25-28], few of their conclusions have been verified by experimental results. Therefore, making experimental observation and quantitative analysis on such buoyant instability can provide more information to help understand the properties of lean hydrogen premixed flames.

Figure 1. Schlieren images of hydrogen-air premixed flames with different equivalent ratios at atmospheric pressure and room temperature.

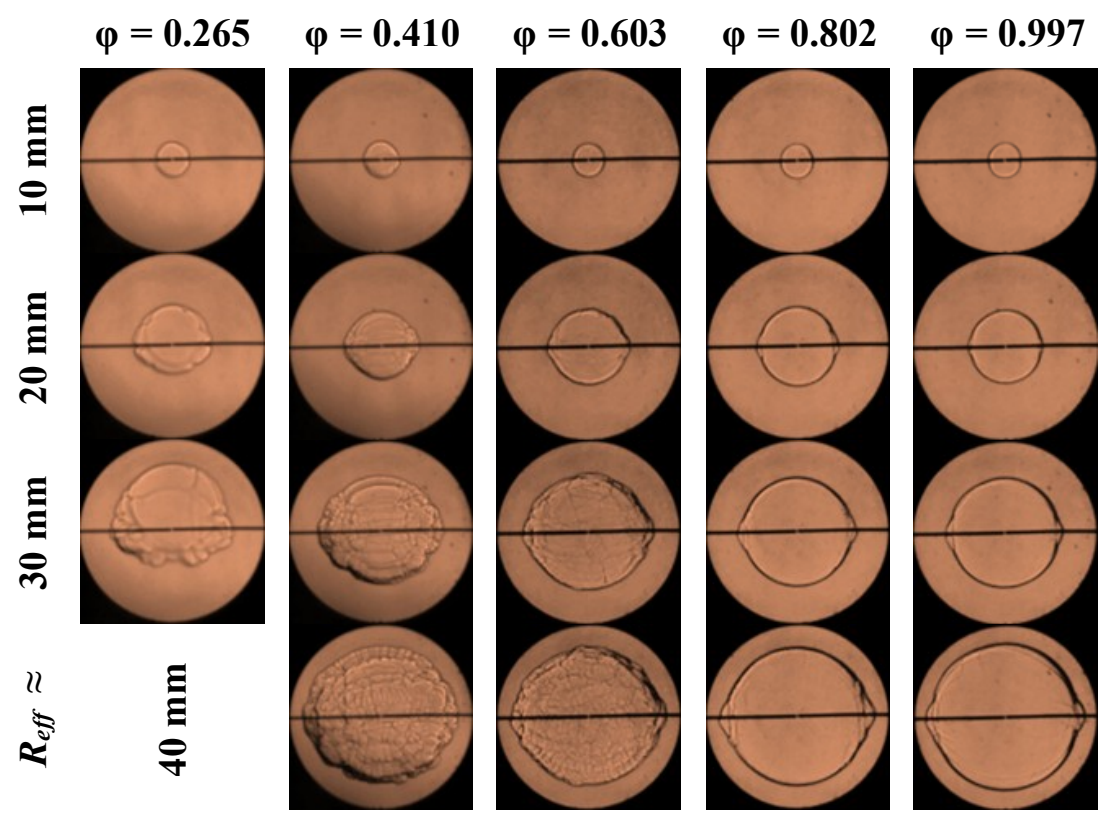

In the present article, the buoyant unstable behavior has been observed experimental in lean premixed hydrogen-air flames within a range of $\varphi$ values from 0.193 to 0.304 , initial pressures $\left(P_{\text {int }}\right)$ from 0.060 MPa to $0.500 \mathrm{MPa}$, and room temperature $(300 \pm 3 \mathrm{~K})$. Two parameters: "float rate" and "critical flame radius" have been proposed to quantitatively investigate the buoyant unstable behavior. Finally, the mechanism of such buoyant unstable behavior has been analyzed and discussed in terms of stretch force and the combined effect of gravity and buoyancy.

\section{Experimental Apparatus and Methodology}

\subsection{Experimental Setup and Procedure}

The experimental rig employed is mainly composed of six parts: a closed combustion vessel, discharge system, ignition system, optical access system, high-speed camera, and data acquisition-control system, as shown in Figure 2. The closed combustion vessel is made of stainless steel and has an inner volume of 2.744 liters. Two quartz windows with an effective diameter of $100 \mathrm{~mm}$ are oppositely mounted in the vessel. The discharge system is designed as a fork type, whose handle is connected to the vessel and four points are respectively connected to the hydrogen bottle, air bottle, vacuum pump, and the environment. 
Figure 2. Schematic diagram of the experimental apparatus.

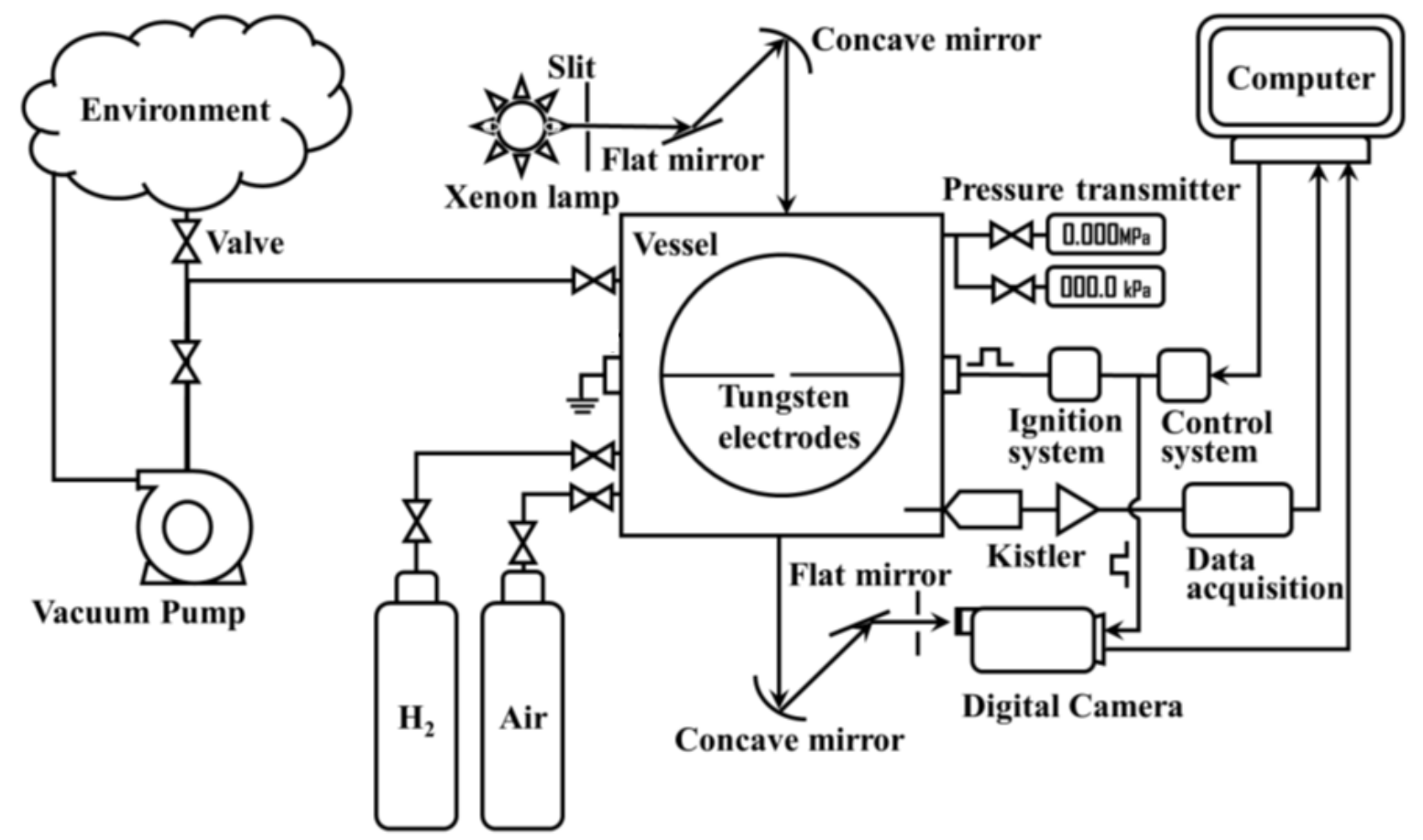

Two electrical pressure transmitters are employed for monitoring the partial pressure during the discharge process, one is a DPA01M-P with a band from $-100.0 \mathrm{kPa}$ to $100.0 \mathrm{kPa}$, and the other is a DPA10M-P with a band from $-0.001 \mathrm{MPa}$ to $+0.999 \mathrm{MPa}$ (both from Taida, Taiwan, China). The ignition system includes a $12 \mathrm{~V}$ transistorized automotive ignition coil, and one pair of tungsten-copper electrodes which are oppositely mounted on the other two sides of the vessel beside the windows. The ignition energy is set as $37 \mathrm{~mJ}$. The optical access system is a Z-shape arranged Schlieren system, which includes a xenon lamp, two flat reflectors, two slits, and two concave mirrors with a diameter of $200 \mathrm{~mm}$ and a focal length of $2000 \mathrm{~mm}$. The high-speed camera is an IDT Motion Pro-Y4 (IDT, Newark, NJ, USA), operating at 10,000 frames per second with a resolution of $256 \times 256$ pixels. The data acquisition-control system is composed of a pressure transducer (6052C, Kistler Instrument AG, Winterhur, Switzerland), a charge amplifier (Kistler 5018A), a data acquisition module (NI PCI-6123, National Instruments, Austin, TX, USA), and a control platform compiled in NI LabVIEW language.

Procedurally, the vessel is evacuated, and then the mixture is prepared according to Dalton's law of additive pressure. The discharge progress can be divided into three stages: (i) filling hydrogen into the vessel until the data shown on the panel of DPA01M-P reaches the desired value, and then evacuating the fork-type pipe after closing Valve A; (ii) releasing air to the pipe after closing Valve $\mathrm{B}$, and then filling air into the vessel after opening Valve A; and (iii) waiting $15 \mathrm{~min}$ to ensuring the homogeneity and quiescence of the mixture. Finally, a control signal is sent to synchronously activate both the ignition system and high-speed camera, and the time delay is set as $4 \mathrm{~ms}$. Prior to refilling for the next set of experiments, the vessel is flushed twice with air to remove any residual products and then it is pumped to vacuum again. It should be noted that absolute vacuum cannot be realized within the vessel, and $-99.7 \mathrm{kPa}$ (shown on the panel of the DPA01M-P) is regarded as a unified signal for the vacuum condition and the residual gas will be accounted for in the volume fraction of air. The hydrogen employed is pure (99.995\%) hydrogen, and the air employed is compressed air. 


\subsection{Analysis of Uncertainties}

Within the present investigation, the uncertainties mainly originate from three issues: (a) the accuracy of equivalence ratio; (b) the clearance effects of the fork-shaped pipe; and (c) the accuracy of the measured flame radius.

The equivalence ratio is controlled by the partial pressure of hydrogen gas and initial pressure, and thus both the accuracies of $\varphi$ and $P_{\text {int }}$ are affected by the truncation errors due to the minimal scale of the pressure transmitters' reading. Within the present discharge system, the uncertainties involves three aspects: (a) the true value of the "prescribed vacuum pressure" $(-99.7 \mathrm{kPa})$; (b) the true value of the amount of filled hydrogen gas; and (c) the true value of the "initial pressure". Taking the case of $\varphi=0.265$ and $P_{\text {int }}=0.06 \mathrm{MPa}$ for an example, the first error source is the truncation error on the reading of "prescribed vacuum, the true value of the set " $-99.7 \mathrm{kPa}$ " falls the range of $-99.74 \mathrm{kPa}$ to $-99.66 \mathrm{kPa}$; the second error source is the truncation error on the reading of pressure transmitter after filling the hydrogen gas, the expectant reading should be "-93.7 $\mathrm{kPa}$ ", but the true value falls the range of $-93.74 \mathrm{kPa}$ to $-93.66 \mathrm{kPa}$, namely, the actual partial pressure of the discharged hydrogen gas falls into the range of $5.92 \mathrm{kPa}$ to $6.08 \mathrm{kPa}$; and the third error source is the truncation error on the reading of the "initial pressure", the true value of the expectant reading " $-40.0 \mathrm{kPa}$ " falls into the range of $-40.04 \mathrm{kPa}$ to $-39.96 \mathrm{kPa}$, namely, the actual partial pressure of the discharge air falls into the range of $53.62 \mathrm{kPa}$ to $53.78 \mathrm{kPa}$; and hence, the actual value of $\varphi$ falls into the range of 0.26209 to 0.269977 , namely, the maximal uncertainties on such case is $1.88 \%$. Figure 3 shows the maximal uncertainties of all the investigated cases within the present article, as can be seen, the maximal uncertainties for the present article is less than $2.00 \%$.

Figure 3. Maximal uncertainties of the cases reported within the present article.

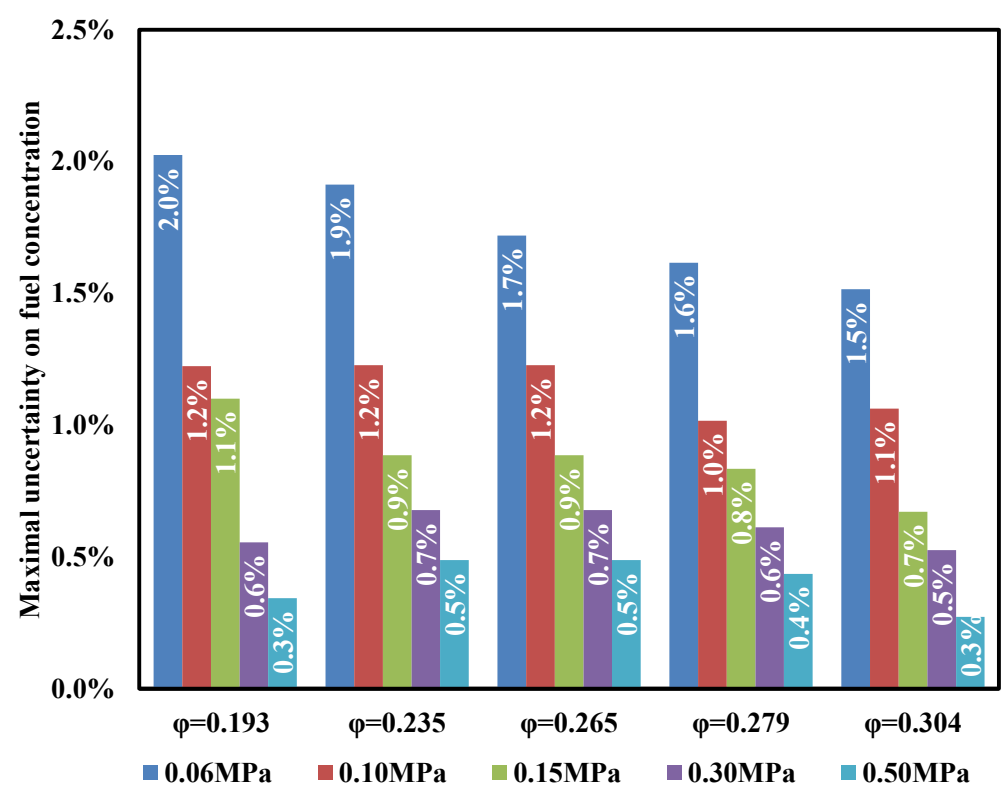

The clearance effects of the fork-shaped pipe can be neglected in the present investigation due to the reasonable discharge approach. In the flame radius measurements, at least five measurements have been performed for each set of conditions to obtain the average values, the repeatability is generally satisfactory, and the uncertainties decrease as the flame radius increases. 


\section{Results and Discussion}

\subsection{Observation of the Morphology of Buoyant Unstable Behavior}

Figure 4 shows the Schlieren images of hydrogen-air premixed flames with different $\varphi$ (from 0.193 to 0.304 ) under atmospheric pressure and room temperature, in which, $R_{\mathrm{sch}}$ is the flame radius of the Schlieren image of the flame front, and it is the calculation result from the flame front's projection area $(A)$ given by:

$$
R_{\mathrm{sch}}=\sqrt{A / \pi}
$$

As can be seen, during the initial period of propagation (within which $R_{\mathrm{sch}} \leq 10 \mathrm{~mm}$ ), nearly all the investigated lean hydrogen premixed flames give a similar propagation behavior in that the flame front propagates outwardly from the ignition position in a spherical manner associated with a smooth surface. For the lean hydrogen premixed flames whose $\varphi$ falls into the range of 0.265 to 0.304 , as flame further propagates, some obvious cracks occur and develop on the flame front (as can be observed on those "relatively richer" lean hydrogen premixed flames shown in Figure 1); but for the lean hydrogen premixed flames with $\varphi=0.193$ and $\varphi=0.235$, the flame front is always smooth during the subsequent propagation.

Figure 4. Schlieren images of hydrogen-air premixed flames with different equivalent ratios at atmospheric pressure and room temperature.

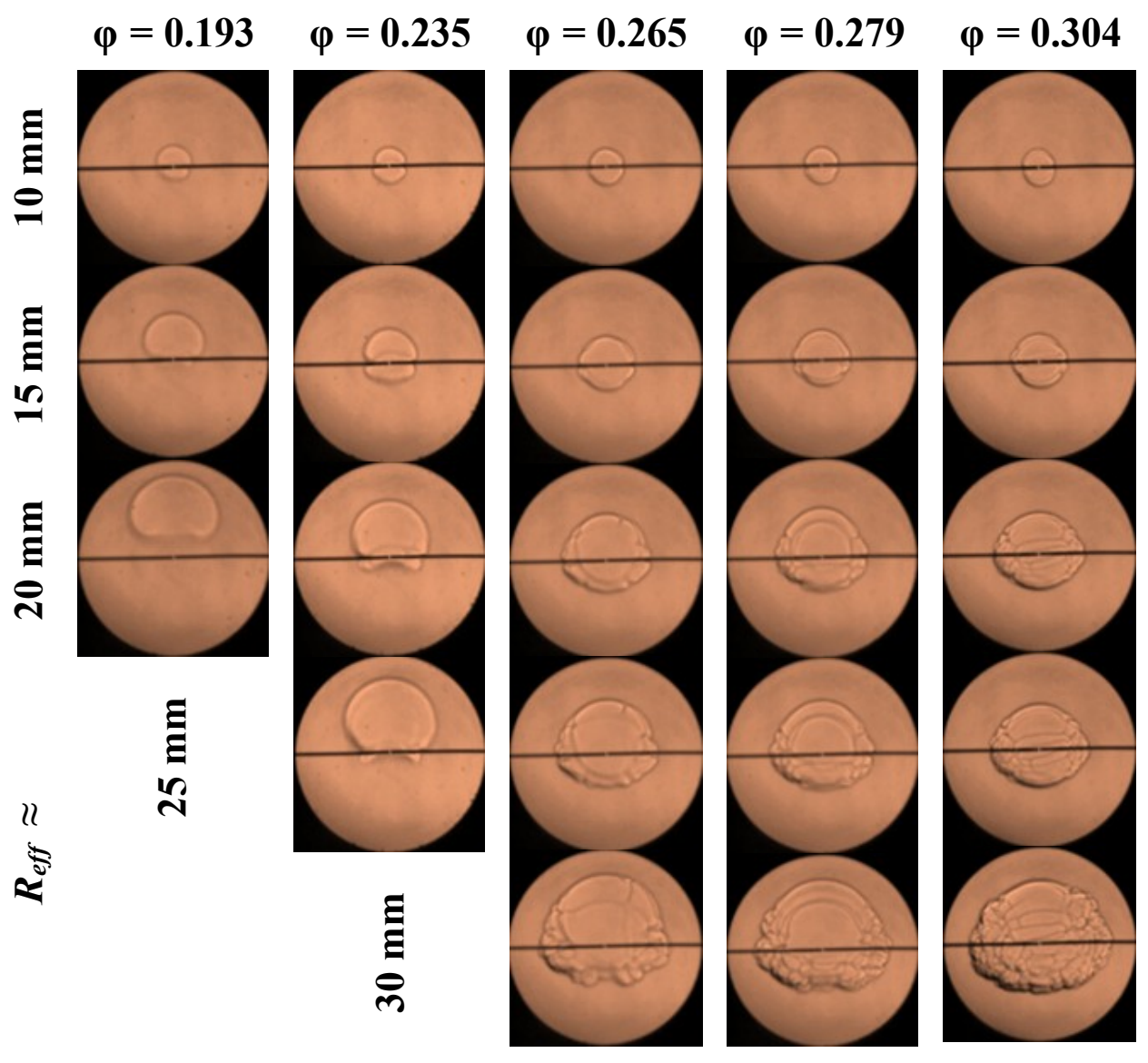


It can be summarized that, for the lean hydrogen premixed flames within the investigated range of fuel concentrations, the "cellularization" of the flamefront has been suppressed with the decrease of fuel concentration, which is distinct from the traditional understanding of the trends of the variation of the cellularization of lean hydrogen premixed flames which is dominated by thermal-diffusive instability.

Accompanying the suppression of cellularization on the flame front, the manner of flame propagation, flame shape, cells' development and cells' distribution are noticeably changed from what is observed in Figure 4. Firstly, regarding the aspect of propagation's manner, the flame has begun to show a tendency of upward propagation when it has propagated to a certain size, and the leaner the flame is, the more obvious the upward tendency becomes, and even lean hydrogen premixed flames with $\varphi=0.193$ and $\varphi=0.235$ have been totally lifted up during the propagation when $R_{\text {sch }} \leq 20 \mathrm{~mm}$. Secondly, on the aspect of flame shape, due to the remarkable upward propagation, the upper part of the flame front has been elongated, and the curvature of the upper part becomes more arc-like while that of the lower partial becomes more flat. Thirdly, on the aspect of cells' development, for those lean hydrogen premixed flames for which cells can also be observed on the flame front, the cells on the lower part still uniformly split into new cells as the flame further propagates, but the cells on the upper part just expand rather than continuously splitting when the flame front has begun to propagate upwards. Fourthly, on the aspect of cells' distribution, for those cellular hydrogen premixed flames, due to the different manner of cell development, the cells' distribution in the upper part is distinct from the uniform distribution on the lower part, and the cells on the upper part are larger in size and less in the number. Since the mentioned four behaviors observed from Figure 4 are distinct from what has always been reported for lean hydrogen premixed flames dominated by thermal-diffusive and hydrodynamic instability, we summarize them as distinct behaviors of buoyant instability, and it also can be concluded that such buoyant instability behaviors are enhanced with the decrease of fuel concentration for lean hydrogen premixed flames under normal pressure and room temperature.

Figure 5 shows the Schlieren images of hydrogen-air premixed flames with $\varphi=0.193$ at room temperature under different $P_{\text {int }}$ conditions. From the comparisons shown in the figure, three phenomena can be easily observed. Firstly, the hydrogen premixed flame with $\varphi=0.193$ will be totally lifted up during the propagation under all the investigated $P_{\text {int }}$ conditions, but the flame still propagates outwardly with a spherical shape within the initial period of propagation under reduced, normal, and certain elevated pressures (such as $0.150 \mathrm{MPa}$ ). Secondly, for a similar flame size, the lift height under reduced and normal pressure are lower than those of flames under elevated pressures; under elevated pressures, the higher the $P_{\text {int }}$ is, the higher the lift height becomes. Thirdly, the flame front is very smooth under reduced and normal pressures, but there exists some obvious "signets" on the flame front under elevated pressures, and the higher the $P_{\text {int }}$ is, the "deeper" the signet can be observed. Based upon the behaviors observed in Figure 5, it seems that the buoyant instability behavior can be enhanced by increasing $P_{\text {int }}$. 
Figure 5. Schlieren images of hydrogen-air premixed flames with $\varphi=0.193$ at different pressures and room temperature.
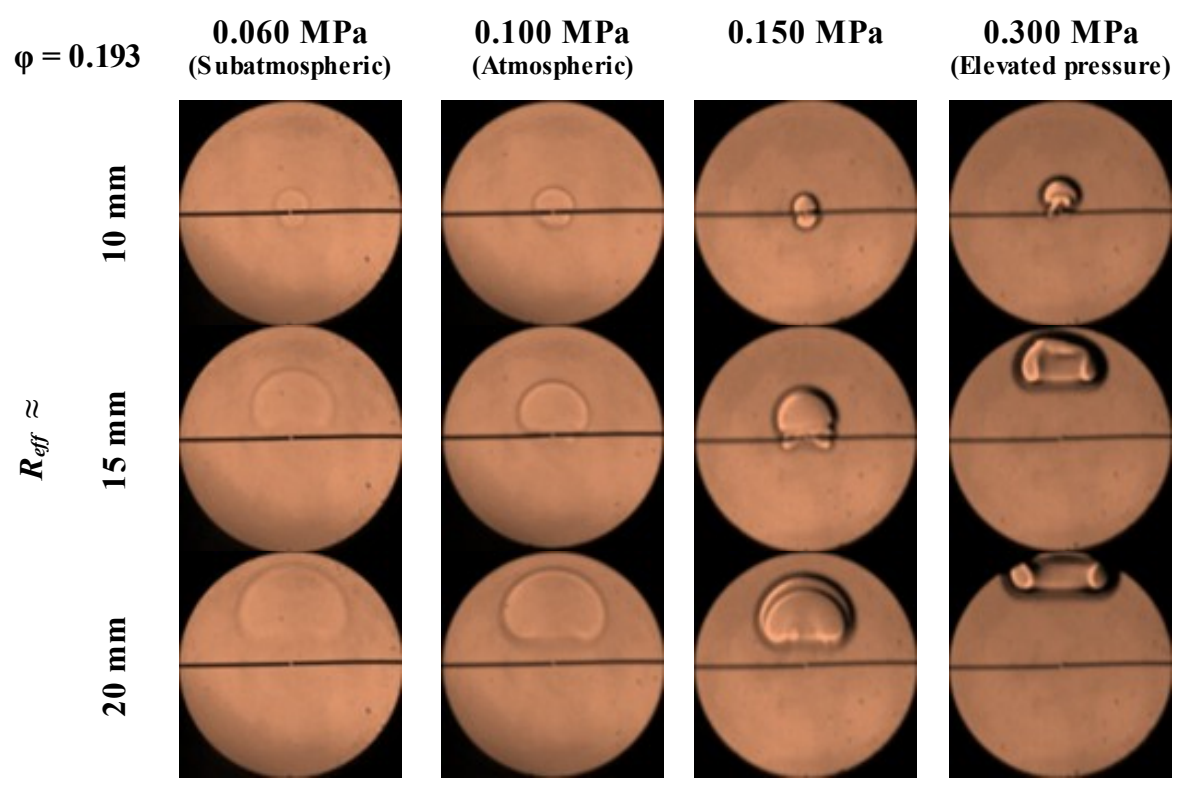

$0.500 \mathrm{MPa}$

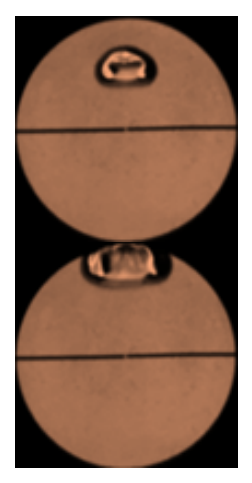

Figure 6 shows the Schlieren images of hydrogen-air premixed flames with $\varphi=0.304$ at room temperature under different $P_{\text {int }}$ conditions. As can be seen, the investigated lean hydrogen premixed flames can maintain the initial spherical outward propagation under elevated pressures, and the upward propagation tendency can just be observed for relatively larger flame sizes under reduced and normal pressure, and the tendency is indeed unremarkable. Under the investigated elevated pressures, the cells formed on the flame front are distributed and develop uniformly, but under reduced and normal pressure, the gradient distribution in size of those cells on the upper part of flame front can still be observed. The higher the $P_{\text {int }}$ is, the more cellular the flame front with a similar flame size becomes, and the less remarkable the tendency of upward propagation becomes, that is, the weaker the buoyant instability behaviors become. Although the observed phenomena are distinct from what is observed in Figure 5, but it is normal considering the traditional understanding of the behavior of lean hydrogen premixed flames. With the absence of buoyant instability for hydrogen premixed flames, increasing $P_{\text {int }}$ will enhance the intensity of the hydrodynamic instability while the thermal-diffusive instability remains unchanged, and the enhanced hydrodynamic instability could enhance the cellularization of flame front; therefore, it seems such phenomena can be easily explained. However, the hydrogen premixed flames with $\varphi=0.193$ under a different $P_{\text {int }}$ also suffer the similar variation trends of hydrodynamic and thermal-diffusive instability, but why do they show distinct phenomena (as shown in Figure 5)? Through the comparisons between the observations on the flame images, it can be found that the two different variation trends of unstable behaviors are accompanied by the distinct phenomenon that the hydrogen premixed flames with $\varphi=0.193$ had been lifted up prior to the formation of cells while a "floating" of the hydrogen premixed flames with $\varphi=0.304$ (if it happened during the propagation) occurs after the formation of cells. Therefore, when the lean hydrogen premixed flames begin to float the trend of such floating is essential to the variation regulations of the unstable behavior of lean hydrogen premixed flames. Therefore, one should make quantitative studies on such buoyant unstable behaviors by examining the "floating" trends of the investigated flames. 
Figure 6. Schlieren images of hydrogen-air premixed flames with $\varphi=0.304$ at different pressures and room temperature.

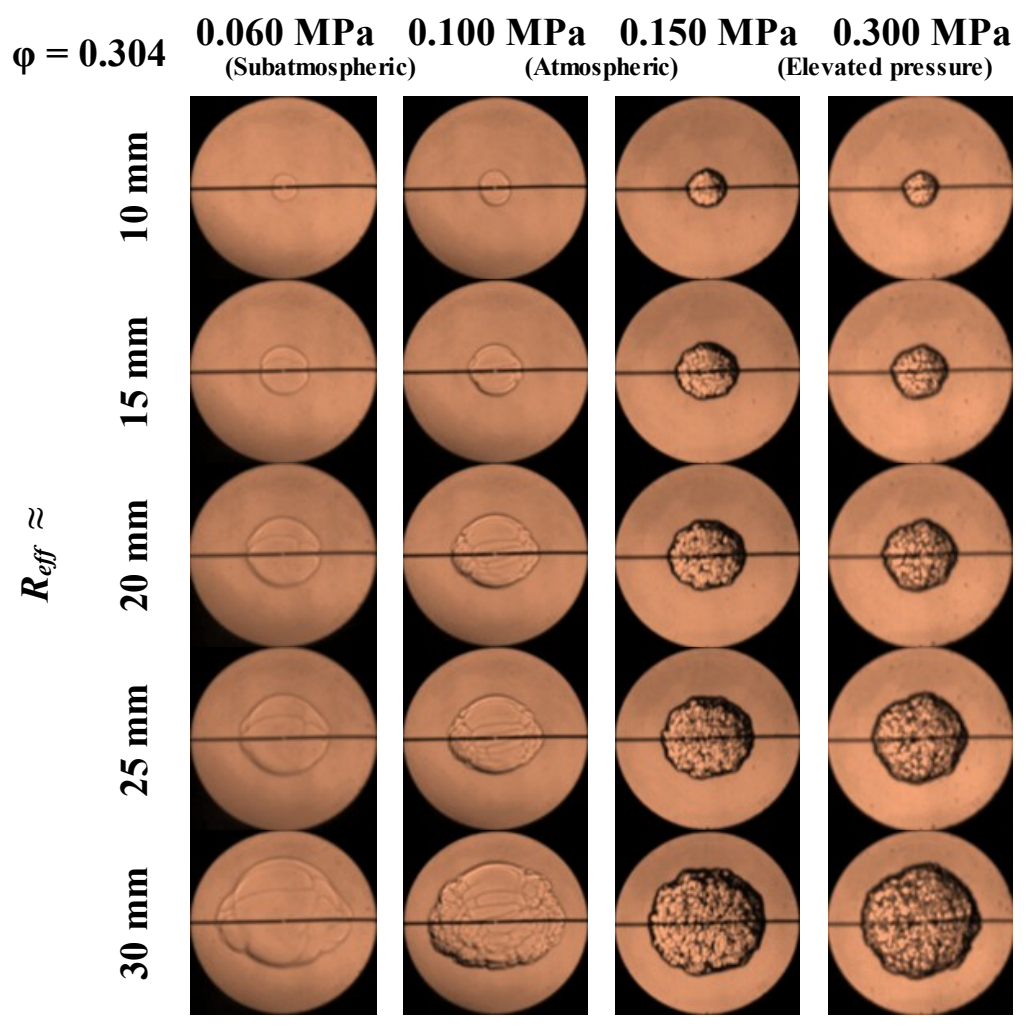

\subsection{Studies on the Buoyant Instability Trends}

Based upon the abovementioned and discussed phenomena, the extent of upward propagation is the most remarkable behavior of the buoyant instability and is also an essential aspect to determine how the unstable buoyant behaviors happen; therefore, it can be taken as the indicator for quantitatively describing the "intensity" of the buoyant instability. Within the present investigation, the extent of upward propagation is indicated by a parameter "Float Rate, $\Psi$ " which is defined as:

$$
\Psi=\Delta L / R_{\text {sch }}=\left(D_{\text {top }}-D_{\text {bottom }}\right) / R_{\text {sch }}
$$

where $D_{\text {top }}$ is the distance from the ignition position to the flame's top; $D_{\text {bottom }}$ is the distance from the ignition to the flame's bottom; and $\Delta L$ is the difference between $D_{\text {top }}$ and $D_{\text {bottom. It can be clearly }}$ understood that, for a specific $R_{\text {sch, }}$ larger the $\Delta L$ is, the more obvious the buoyant instability behavior becomes; while, for a specific $\Delta L$, the smaller the $R_{\text {sch }}$ is, the more obvious the buoyant instabilty; therefore, the parameter "float rate" provides a good comprehensive characterization of the buoyant instability.

Figure 7 shows the variation of $\Psi$ with $R_{\text {sch }}$ under different conditions. As can be seen, under all the investigated conditions, the value of $\Psi$ increases as $R_{\text {sch }}$ increases, namely, such buoyant instability will be continuously enhanced as flame expands once the buoyant instability has ocurred, which means the buoyant instability behaviors have a self-enhancing nature. From Figure 7a, it can be found that, during the period of constant pressure, $\Psi$ increases as the flame expands with an accelerating growth rate, and the rate of acceleration at a specific $R_{\text {sch }}$ is increased with the reduction of $\varphi$, which indicates that the leaner the hydrogen premixed flame is, the stronger the buoyant instability becomes. When the 
flame front enters a zone with an increasing pressure, $\Psi$ still increases as the flame expands but the growth rate of $\Psi$ the decreasing noticeably.

Figure 7. Variation of Float Rate with flame radius: (a) with different equivalence ratios under atmospheric pressure; $(\mathbf{b})$ with $\varphi=0.193$ under different initial pressures; (c) with $\varphi=0.265$ under different initial pressures; and (d) with $\varphi=0.304$ under different initial pressures.

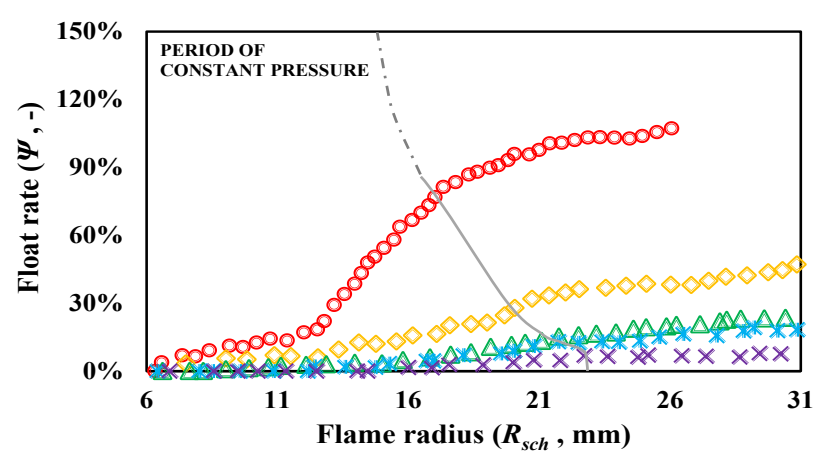

$\bigcirc \varphi=0.193 \diamond \varphi=0.235 \Delta \varphi=0.265 * \varphi=0.279 \times \varphi=0.304$

(a)

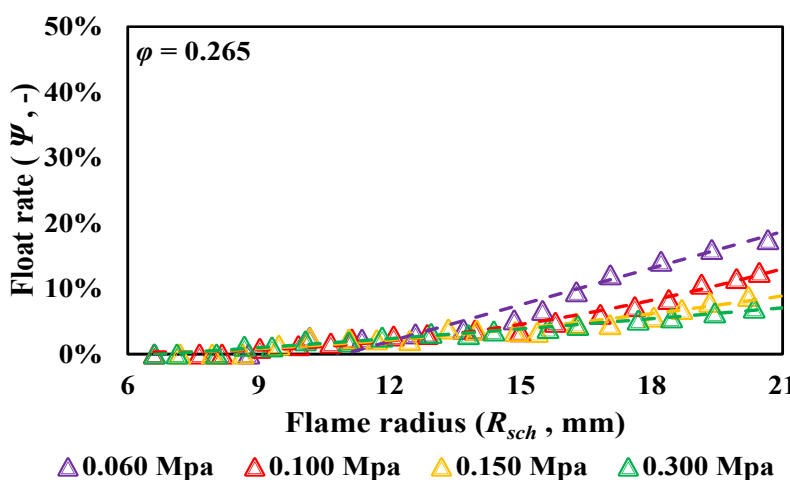

(c)

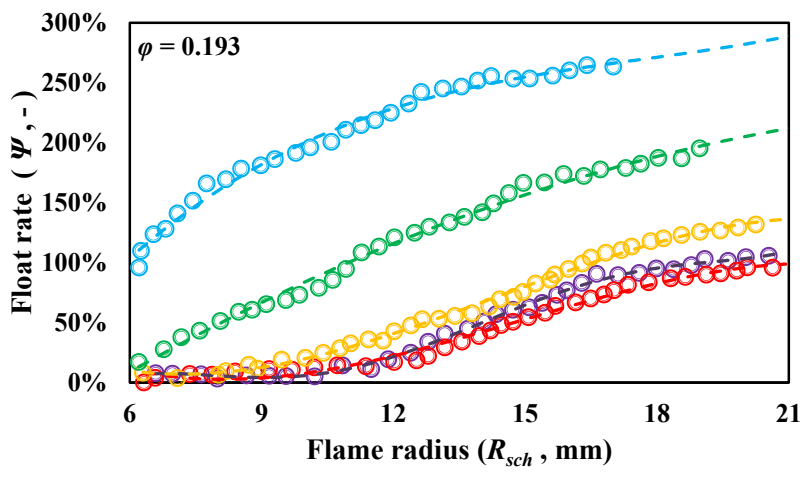

$\bigcirc 0.060$ Мра $\bigcirc 0.100$ Мра $\odot .150$ Мра $\bigcirc 0.300$ Мра $\bigcirc 0.500$ Мра

(b)

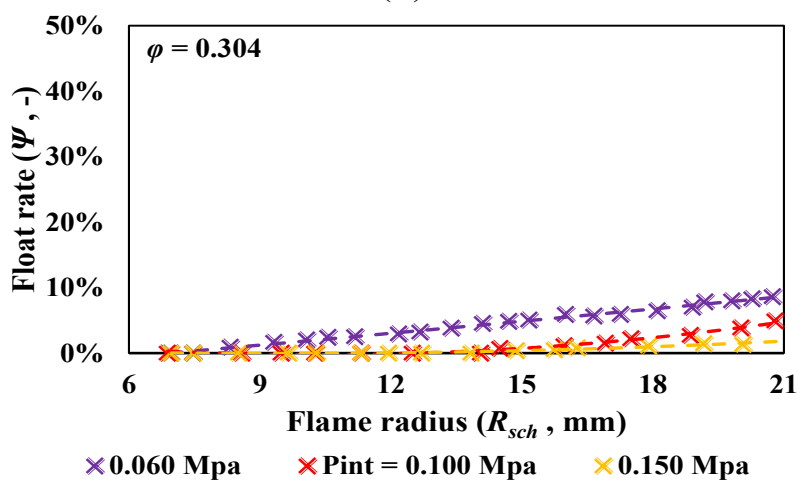

(d)

Compared to the influences of $\varphi$ on the buoyant instability, the influences of $P_{\text {int }}$ on the variation trends of $\Psi$ are relative complex. As can be seen from Figure $7 \mathrm{~b}$, for the lean hydrogen premixed flame with $\varphi=0.193$, as $P_{\text {int }}$ increases, $\Psi$ at a similar $R_{\text {sch }}$ firstly decreases and then increases, and the minimal value is attained at atmospheric pressure; which indicates the variation of $P_{\text {int }}$ (relative to atmospheric pressure) could enhance the intensity of the buoyant instability. For the lean hydrogen premixed flames with $\varphi=0.265$ and $\varphi=0.304$, the values of $\Psi$ at a specific $R_{\text {sch }}$ monotonously increases as $P_{\text {int }}$ increases. Furthermore, no matter whether promoting or restraining, the effects of $P_{\text {int }}$ on the variation of $\Psi$ become weaker as $\varphi$ increases.

As mentioned above and discussed in the previous subsections, when the lean hydrogen premixed flames have begun to be lifted up is essential to the variation trends of the buoyant instability behavior; therefore, we propose the term "critical flame radius, $R_{\mathrm{cr}}$ " (which is defined as the flame radius at which $\Psi$ has increased up to $10 \%$ ) as the indicator to the onset of the buoyant instability. As shown in Figure 8 , for the lean hydrogen premixed flames with $\varphi \leq 0.235, R_{\mathrm{cr}}$ generally decreases as $P_{\text {int }}$ increases, and the extent of decrease is enlarged as $P_{\text {int }}$ increases for a specific $\varphi$, and the variation 
extent decreases as $\varphi$ increases. For the flames with $\varphi \geq 0.265, R_{\mathrm{cr}}$ increases as $P_{\text {int }}$ increases, the extent of $R_{\mathrm{cr}}$ varies noticeably with the increase of $P_{\text {int }}$ for a specific $\varphi$ and/or the increase of $\varphi$ for a specific $P_{\text {int. }}$ Furthermore, combined with the observed flame images, for the lean hydrogen premixed flames with $\varphi=0.193$ and $\varphi=0.235, R_{\text {cr }}$ is less than the flame radius at which cells can be observed on the flame front (actually, for most investigated cases in the present article, no cells have been formed on those flames while the flame was still propagating within the field of view); for the lean hydrogen premixed flames with $\varphi=0.265, \varphi=0.279$ and $\varphi=0.304, R_{\mathrm{cr}}$ is larger than the flame radius at which cells have been formed.

Figure 8. Critical flame radius effect on the buoyant instability for hydrogen-air premixed flames with initial pressure for different equivalence ratios.

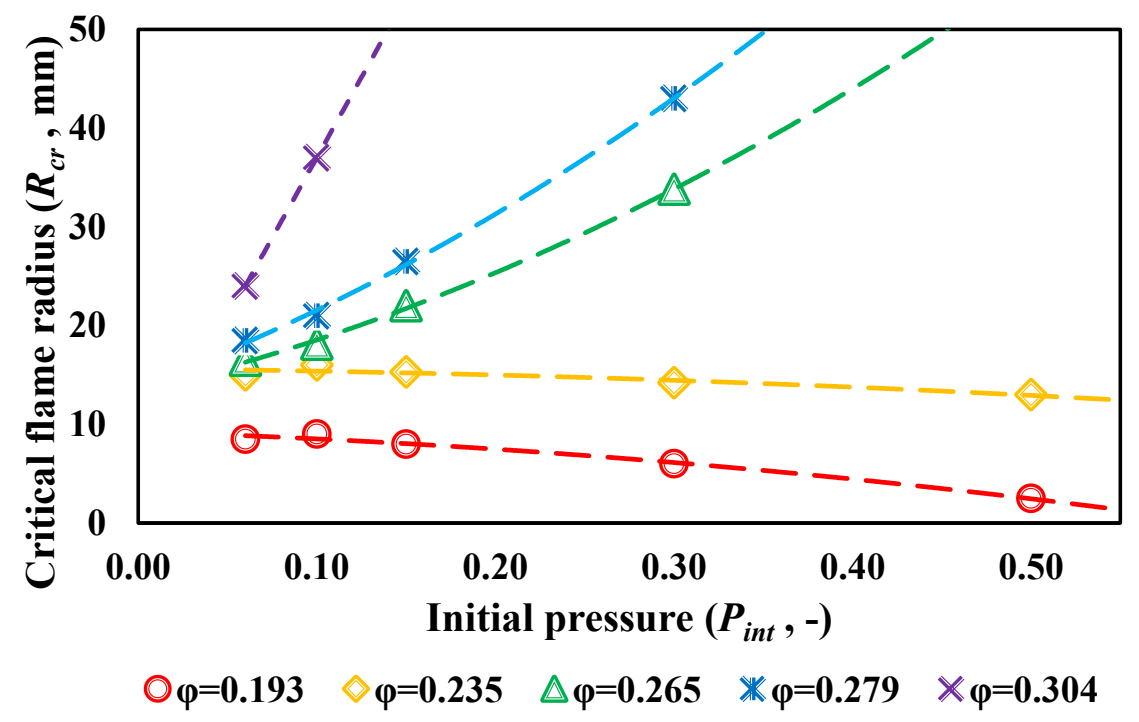

Based upon the observation and trends mentioned above, it can be supposed that decreasing $\varphi$ could promote the buoyant instability under a specific $P_{\text {int }}$, but the effects of $P_{\text {int }}$ on the buoyant instability are different for different $\varphi$ values.

\subsection{Discussion on the Dominating Role of the Buoyant Instability}

An outwardly propagating flame mainly suffers three forces, besides its own thermal expansion, which are respectively the stretch force, buoyancy, and gravity, as shown in Figure 9. For a spherical outwardly propagating flame, in the absence of the influence of electrodes, the stretch force upon the right semi-part should be equal to that upon the left semi-part due to the symmetrical structure, and thus the flame movement in the vertical direction is dominated by the combined effect of buoyancy and gravity. It should be noted that the differences in geometric parameters (including the length, radius, roughness, etc.) and physical characteristics (such as materials, carbon laydown on the surface of electrodes) of electrodes can induce differences in the stretch forces on the right and left semi-parts, and these unbalanced stretch forces on both semi-parts can be observed from the flame shape if such differences are sufficiently large. Due to the good symmetrical characteristics observed in the flame images, such differences were neglected in the present article. 
Figure 9. Schematic diagram of the forces acting upon a spherical propagating flame.
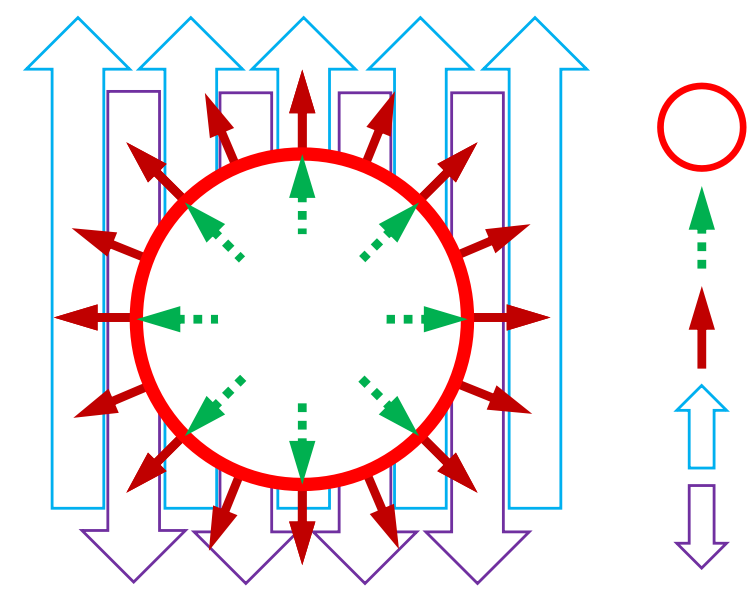

Flamefront

Thermal expansion

Global stretch

Buoyant field

Gravity field

According to the Archimedes' principle, the buoyancy of a flame is the product of gravity acceleration, the flame's volume, and the unburned reactants' density; therefore, the combined effect of buoyancy and gravity can be indicated by the flame's volume and the density difference across the flame front ( $\Delta \rho$, the difference on the density between the unburned and the burned fuel). If we consider combustion occurs at an adiabatic temperature and composition at constant pressure, the value of $\Delta \rho$ is a constant value during the flame's propagation, and hence the global result of the competition between buoyancy and gravity is being increased during the propagation due to the flame's increasing volume, which explains well why $\Psi$ increases as $R_{\text {sch }}$ increases under any conditions.

Figure 10. Density difference of hydrogen-air premixed flames with equivalence ratio under different initial pressures.

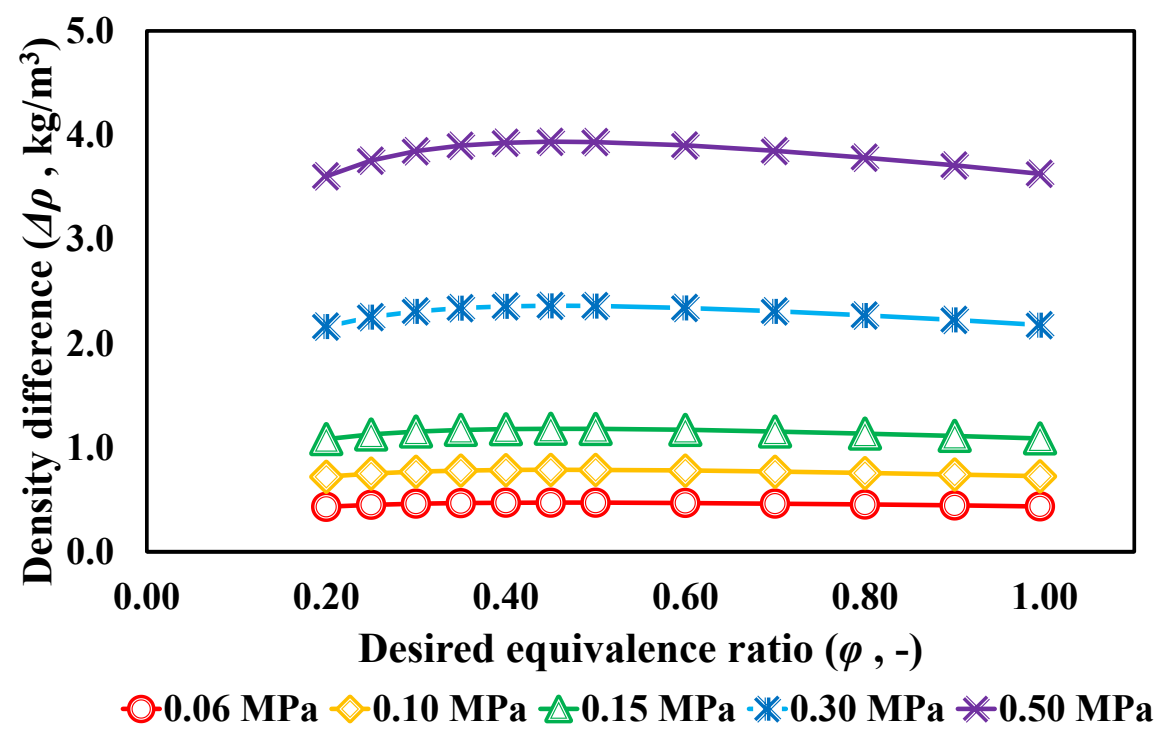

Figure 10 shows the variation trend of $\Delta \rho$ with $\varphi$ under different $P_{\text {int, }}$ where both the densities of unburned and burned mixtures are calculated using the chemical equilibrium program GASEQ [29]. As can be seen, for lean hydrogen premixed flames, with the increase of $\varphi$, the value of $\Delta \rho$ firstly increases and then decreases, and the maximal value is attained near $\varphi=0.4$, but the difference in $\Delta \rho$ 
can hardly be observed when $P_{\text {int }}$ is no more than $0.15 \mathrm{MPa}$. The calculated results indicate that, for the case of $P_{\text {int }} \leq 0.15 \mathrm{MPa}$, the global result of the competition between buoyancy and gravity is similar for different $\varphi$ values, that is, the effects of such competition hardly induce any obvious difference as the fuel concentration varies; while, for the case of $0.30 \mathrm{MPa} \leq P_{\text {int }} \leq 0.50 \mathrm{MPa}$, the competition between buoyancy and gravity will be weakened when the fuel concentration becomes much leaner than 0.4. Furthermore, for a specific $\varphi$, the higher the $P_{\text {int }}$ is, and the larger the value of $\Delta \rho$ becomes, which means the global result of the competition between buoyancy and gravity should be enhanced.

However, just some cases (in which $\varphi \leq 0.235$ ) in our investigation can be explained well by the variation trend, and the others (in which $0.265 \leq \varphi$ ) still cannot be explained by solely considering the influence of $\Delta \rho$. Therefore, other factor(s) must exist that affect the buoyant instability.

Actually, as a result of the competition between buoyancy and gravity, the global stretch forces on both semi-parts of the flame in the gravity direction can hardly be absolutely balanced; as observed from Figures 1, 4, and 5, the flame curvature of the upper part of the flame front is distinct from that of the lower part. For provide further information about the unstable premixed flames, the global stretch forces acting upon the flames have been analyzed quantitatively using the "stretch rate" $(\alpha)$, which reflects the intensity of the stretch effects by the variation trend of the flame fronts' area, is defined as the Lagrangian time derivative of the logarithm of the area of flame, as given in [30]:

$$
\alpha=\mathrm{d}(\ln A) / \mathrm{d} t=(1 / A) /(\mathrm{d} A / \mathrm{d} t)
$$

where $A$ is the area of the projected flame's image. Figure 11 shows the variation of global stretch rate with flame radius under different fuel concentrations and initial pressures. As can be seen from Figure $11 \mathrm{a}$, under normal pressure and room temperature, as $R_{\text {sch }}$ increases, $\alpha$ decreases towards a convergent value rather than zero as the interference between the flame and the unburned reactants won't vanish, the value of $\alpha$ at a same $R_{\text {sch }}$ decreases as $\varphi$ decreases. The results indicate that the global stretch force acting upon the flame declines towards a certain level as the flame expands for a specific lean hydrogen premixed flame, and the global stretch force is weakened with the decrease of fuel concentration under normal pressure. Actually, during the initial period of propagation, the flame's volume is very small, and the result of the competition between buoyancy and gravity is correspondingly small, but the stretch force on the flame is the largest for the whole propagation; however, as the flame propagates further, the result of the competition between buoyancy and gravity is being enlarged due to the flame's increasing volume, while the stretch force on the flame is declining remarkably. Without consideration of any other external forces, a strong result of the competition between buoyancy and gravity will drive the flame upwards, and the stronger such competition is, the more obvious the upward propagation will become; with the absence of buoyancy effects, a strong stretch can kept the flame front smooth and propagating outwardly with the suppression of the unstable behaviors [31-33]. Based upon the observed unstable behaviors and the mentioned analysis, it can be said that the initial spherical propagation of lean hydrogen premixed flames is dominated by the strong global stretch force rather than the weak competition between buoyancy and gravity, and the later upward propagation of the lean hydrogen premixed flame is caused by the sufficiently strong competition between buoyancy and gravity. 
Figure 11. Global stretch rate of hydrogen-air premixed flames with flame radius: (a) with different equivalence ratios under atmospheric pressure; (b) with $\varphi=0.193$ under different initial pressures; and (c) with $\varphi=0.304$ under different initial pressures.
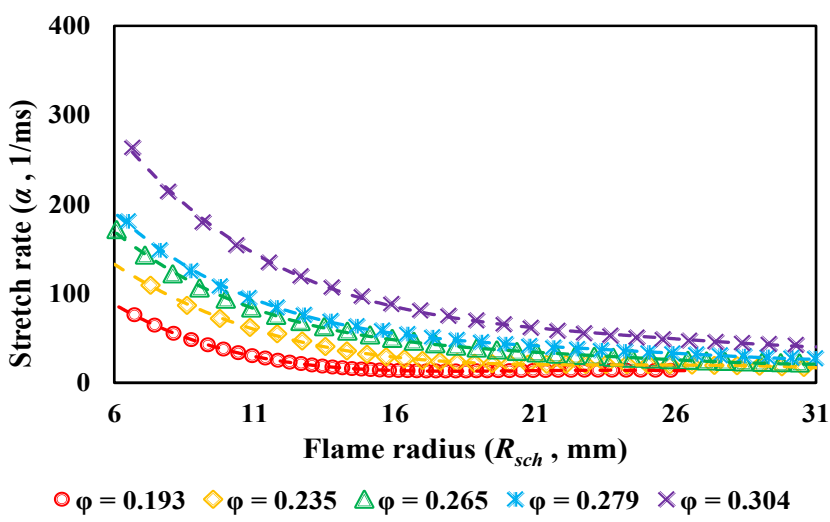

(a)

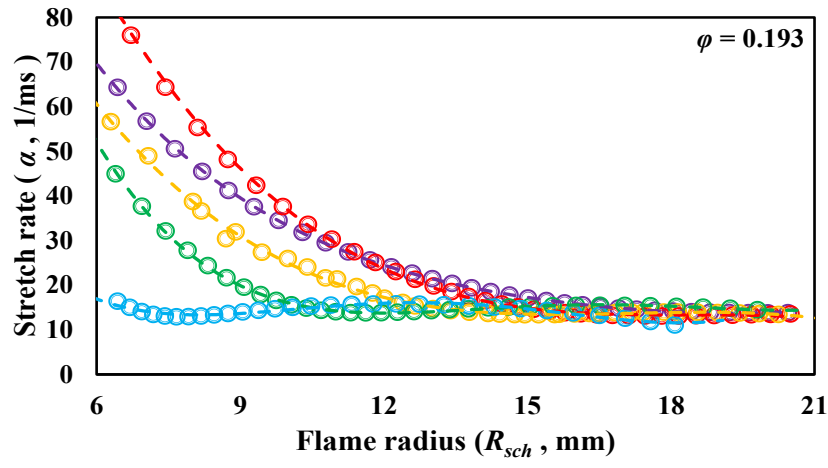

$\bigcirc 0.060$ Мра $\bigcirc 0.100$ Мра $\bigcirc .150$ Мра $\bigcirc 0.300$ Мра $\bigcirc 0.500$ Мра

(b)

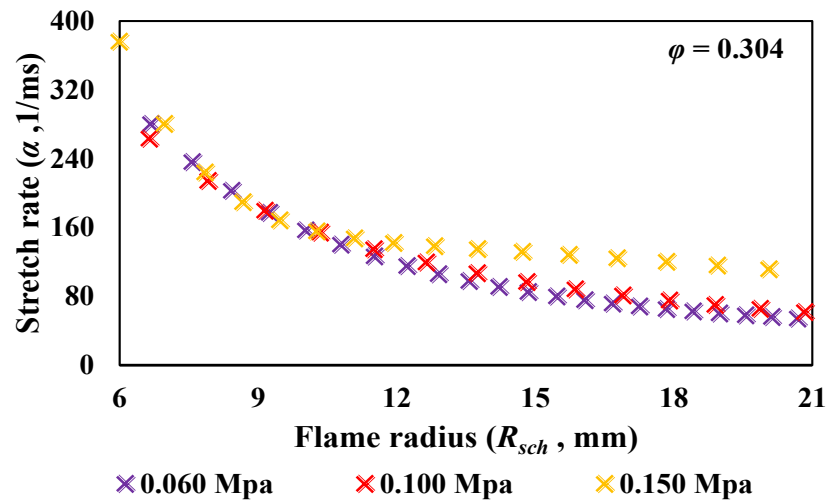

(c)

From Figure 11b, it can be found that, for the hydrogen-air premixed flames with $\varphi=0.193$, the values of $\alpha$ at a same $R_{\text {sch }}$ are distinct under different $P_{\text {int }}$ during the initial period of propagation, but such differences decline noticeably as flame propagates further, and the difference can even be neglected when $R_{\text {sch }}$ is more than $15 \mathrm{~mm}$. For the initial period of propagation, as $P_{\text {int }}$ increases within the investigated range, $\alpha$ at a same $R_{\text {sch }}$ firstly increases and then decreases, and the maximal value is attained at atmospheric pressure. The results indicate that for such lean hydrogen premixed flames under normal and elevated pressures, the higher the $P_{\text {int }}$ is, the weaker the intensity of the global stretch force becomes; furthermore, the competition between buoyancy and gravity is enhanced (as reported in 
Figure 10). Therefore, the intensity of the competition between buoyancy and gravity may exceed the intensity of the global stretch force, and such a difference will be enhanced with the increase of $P_{\text {int }}$. Under reduced pressure, both the intensities of the global stretch force and the competition between buoyancy and gravity are weakened compared to the normal pressure, but the difference between the two aspects may also weaken, which means the composite effects of such a difference may be stronger than those under normal pressures; hence, the buoyant instability behavior would be enhanced compared to that seen under normal pressure. Such conclusions can better explain the buoyant instability behaviors observed in the present article for the lean hydrogen premixed flames with $\varphi=0.193$ and $\varphi=0.235$ (which have similar variation trends for both $\Delta \rho$ and $\alpha$ ).

From Figure 11c, it can be found that for the premixed hydrogen-air flames with $\varphi=0.304$, the value of $\alpha$ at a same $R_{\text {sch }}$ increases monotonously as $P_{\text {int }}$ increases within the investigated range, the difference on $\alpha$ is relatively smaller during the initial propagation period, but such a difference increases remarkably as the flame propagates further. For the initial period of propagation, the difference of $\alpha$ is not obvious under different $P_{\text {int }}$ values, which means verifying the initial pressure won't induce any noticeable variation of the global stretch force acting on the flame front; however, the competition between buoyancy and gravity will be enhanced by increasing $P_{\text {int, }}$ namely, the buoyant instability potential has been enhanced. However, from the flame images shown in Figure 6, few related phenomena can be observed. Actually, by making a comparison between the cases of $\varphi=0.193$ and $\varphi=0.304$, it can be found that the influence of $P_{\text {int }}$ on the value of $\Delta \rho$ is highly similar (shown in Figure 10), namely, the competition between buoyancy and gravity under a specific $P_{\text {int }}$ is mostly kept at a similar level; however, the values of $\alpha$ at a same $R_{\text {sch }}$ are distinct, the minimal value of $\alpha$ for the case of $\varphi=0.304$ is even higher than the maximal value of the case of $\varphi=0.193$ for a specific $P_{\text {int }}$, that is, the intensity of the global stretch force on the flame front has been significantly enhanced. Therefore, it can be said that for lean hydrogen premixed flames with $\varphi=0.304$, increasing $P_{\text {int }}$ induces an obvious enhancement of the global stretch force rather than the competition between buoyancy and gravity, and the role of global stretch force on maintaining the flame spherically outwardly dominates the effect of the competition between buoyancy and gravity driving the flame upwards, and hence the flame will not display any obvious buoyant instability behaviors. However, when the flame has propagated to a certain size, the increased competition between buoyancy and gravity (induced by the flame's increasing volume) will exceed the declining global stretch force, and then the tendency of upward propagation will appear again (as shown in Figure 6). Figure 12 shows the variation trend of flow rate with global stretch rate under different conditions. As can be seen, the flow rate will not be observed when the stretch rate is still sufficiently large, and the value will be remarkably increased when the stretch rate further decreases from certain smaller values. Comparing the cases of lean hydrogen premixed flames with $\varphi=0.193$ and with $\varphi=0.304$, it can be seen that under a specific $P_{\text {int }}$, the value of $\Psi$ for the leaner flame is lower than that for the richer, which is similar to the correlation of $\Delta \rho$. The results again indicate that the buoyant instability is related to $\Delta \rho$ (the competition between buoyancy and gravity) and $\alpha$ (the global stretch force). 
Figure 12. Flow rate of hydrogen-air premixed flames with global stretch rate: (a) with different equivalence ratios under atmospheric pressure; (b) with $\varphi=0.193$ and $\varphi=0.304$ under different initial pressures.

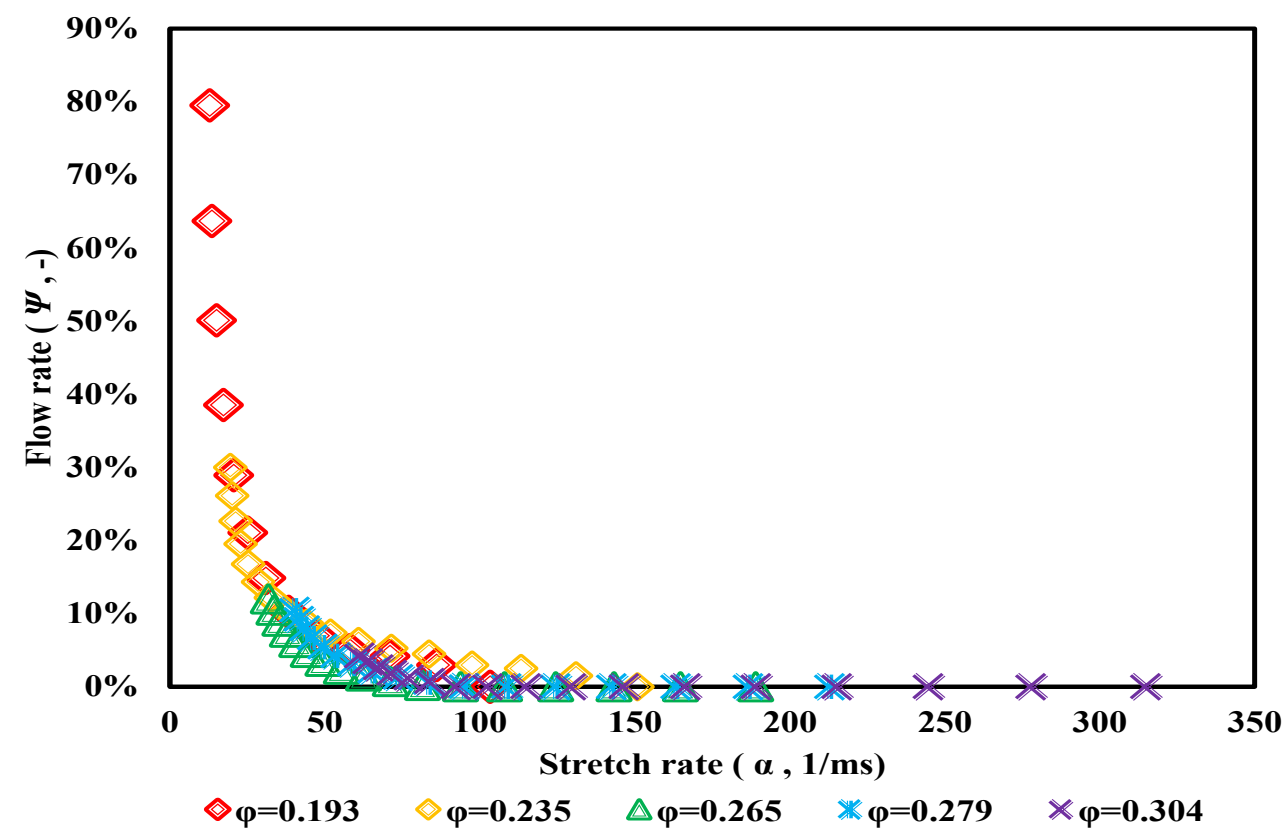

(a)

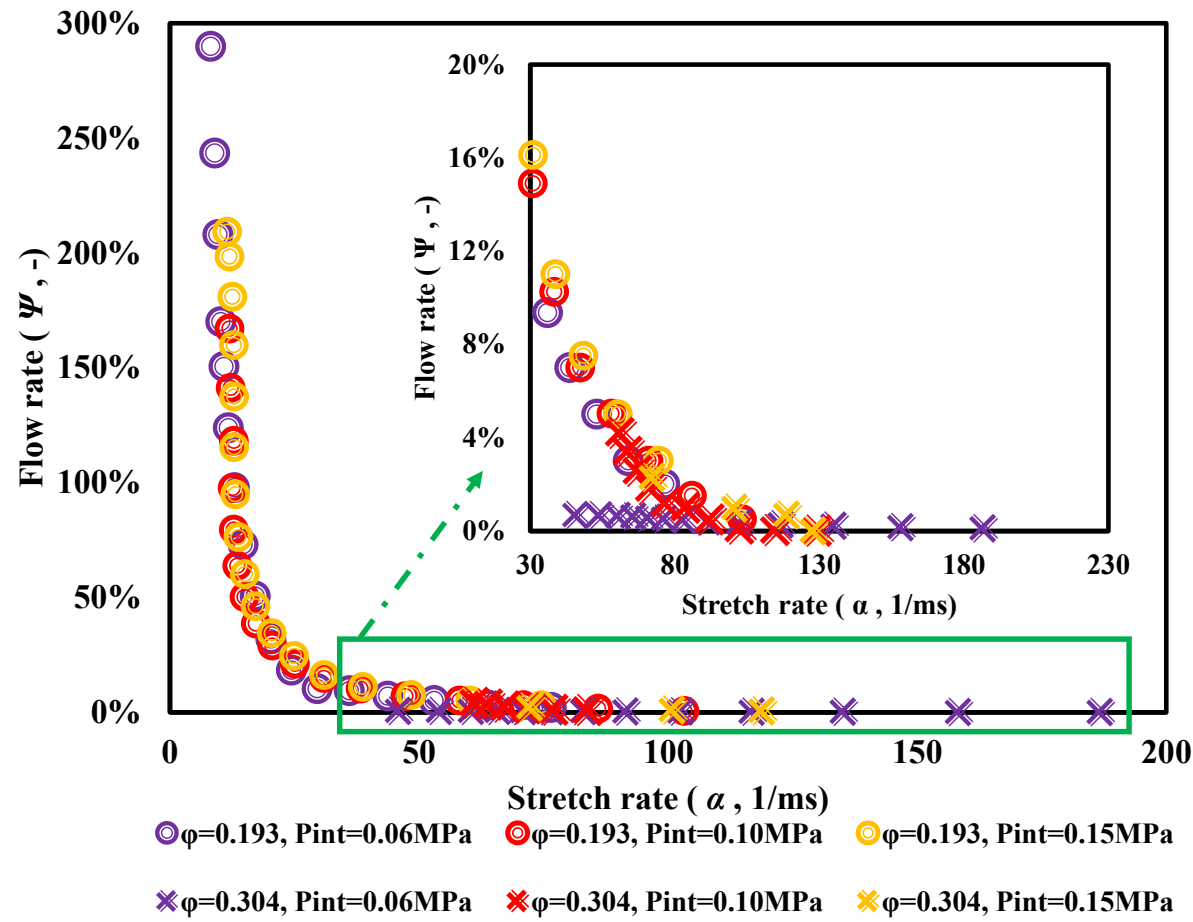

(b)

Based upon the above discussions, it can be concluded that whether the buoyant instability can be seen or not is determined by the result of the competition between the stretch force acting on the flame and the combined resultant of buoyancy and gravity, and the flame won't show an obvious buoyant instability if the stretch force plays a dominant role in the propagation; otherwise, the flame will propagate upwards. 


\section{Conclusions}

The buoyant instability behavior of lean premixed hydrogen-air flames has been observed and investigated in a closed combustion vessel under a range of equivalence ratios (from 0.193 to 0.304 ), initial pressures (from $0.060 \mathrm{MPa}$ to $0.500 \mathrm{MPa}$, in a term of absolute pressure), and room temperature. The main conclusions can be summarized as follows:

(1) Under atmospheric pressure, the flame front of a lean hydrogen premixed flames with $\varphi \leq 0.304$ is smoother than those with richer fuel concentrations, and the leaner the fuel concentration is, the much smoother the flame front with a similar size becomes; such a variation trend of the flame front is distinct from the traditional understanding of the cellular instability of lean hydrogen premixed flames;

(2) Accompanying the smoothening of the flame front, with the further decrease of fuel concentration, the flame shows a tendency to propagate upwards rather than maintaining the initial spherical outward propagation, and the geometric center of those lean hydrogen premixed flames will be lifted up during the propagation, namely, those flames show buoyant instability behavior;

(3) To quantitatively analyze the variation trends of such buoyant instability behaviors, two parameters, "flow rate" and "critical flame radius", have been proposed;

(4) Under a specific pressure, the critical flame radius will be decreased with the decease of $\varphi$, and the float rate of upwardly propagating flames of a similar size will be increased, namely, the buoyant instability behavior will be enhanced with the decline of fuel concentration;

(5) With a specific $\varphi$, the variation trends of float rate and critical flame radius induced by the variation of $P_{\text {int }}$ are different for different $\varphi$; in terms of leanness for hydrogen-air premixed flames with $\varphi \leq 0.235$, increasing $P_{\text {int }}$ will induce a decline of critical flame radius and the growth of the float rate for the same flame size, namely, the buoyant instability behaviors are enhanced; however, in terms of the lean hydrogen-air premixed flames with $\varphi \geq 0.265$, an inverse variation trend will be observed;

(6) Such buoyant instability behaviors are determined by both the global stretch force and the competition between buoyancy and gravity, just when the intensity of the former is less than that of the latter, with a lean hydrogen premixed flame show buoyant instability, otherwise the lean hydrogen premixed flame will maintain a spherical shape and propagate outwards.

\section{Acknowledgments}

The present investigation is primarily supported by the Ph.D Programs Foundation of Ministry of Education of China (No. 20110009110023), the Fundamental Research Funds for the Central Universities (No. M11JB00300), and the Postdoctoral Science Foundation of Beijing Jiaotong University (No. 98930098).

\section{Author Contributions}

The author contributions were as follows: Zuo-Yu Sun and Guo-Xiu Li are the guarantors of the integrity of entire study and are responsible for the study concepts and study design. They also 
performed the literature search, the data analysis/interpretation and the statistical analysis. All the authors participated in the experimental studies and the data acquisition. Zuo-Yu Sun defined the intellectual content of and prepared and edited the manuscript. The manuscript was revised/reviewed by Zuo-Yu Sun And Guo-Xiu Li and the final approval was given by Guo-Xiu Li.

\section{Conflicts of Interest}

The authors declare no conflict of interest.

\section{References}

1. Karim, G.A. Hydrogen as a spark ignition engine fuel. Int. J. Hydrog. Energy 2003, 28, 569-577.

2. Pudukudy, M.; Yaakob, Z.; Mohammad, M.; Narayanan, B.; Sopian, K. Renewable hydrogen economy in Asia-Opportunities and challenges: A review. Renew. Sustain. Energy Rev. 2014, 30, 743-757.

3. Sun, Z.Y.; Liu, F.S.; Liu, X.H.; Sun, B.G.; Sun, D.W. Research and development of hydrogen fuelled engines in China. Int. J. Hydrog. Energy 2012, 37, 664-681.

4. Fayaz, H.; Saidur, R.; Razali, N.; Anuar, F.S.; Saleman, A.R.; Islam, M.R. An overview of hydrogen as a vehicle fuel. Renew. Sustain. Energy Rev. 2012, 16, 5511-5528.

5. Verhelst, S. Recent progress in the use of hydrogen as a fuel for internal combustion engines. Int. J. Hydrog. Energy 2014, 39, 1071-1085.

6. Hu, E.; Huang, Z.; Liu, B.; Zheng, J.; Gu, X. Experimental study on combustion characteristics of a spark-ignition engine fueled with natural gas-hydrogen blends combining with EGR. Int. J. Hydrog. Energy 2009, 34, 1035-1044.

7. Eisazadeh-Far, K.; Moghaddas, A.; Rahin, F.; Metghalchi, H. Burning speed and entropy production calculation of a transient expanding spherical laminar flame using thermodynamic model. Entropy 2010, 12, 2485-2496.

8. Sun, Z.Y.; Liu, F.S.; Bao, X.C.; Liu, X.H. Research on cellular instabilities in outwardly propagating spherical hydrogen-air flames. Int. J. Hydrog. Energy 2012, 37, 7889-7899.

9. Matalon, M. Intrinsic flame instabilities in premixed and nonpremixed combustion. Annu. Rev. Fluid Mech. 2007, 39, 163-191.

10. Mitani, T.; Williams, F.A. Studies of cellular flames in hydrogen-oxygen-nitrogen mixtures. Combust. Flame 1980, 39, 169-190.

11. Kadowaki, S.; Suzuki, H.; Kobayashi, H. The unstable behavior of cellular premixed flames induced by intrinsic instability. Proc. Combust. Inst. 2005, 30, 169-176.

12. Vu, T.M.; Park, J.; Kim, J.S.; Kwon, O.B.; Yun, J.H. Experimental study on cellular instabilities in hydrocarbon/hydrogen/carbon monoxide-air premixed flames. Int. J. Hydrog. Energy 2011, 36, 6914-6924.

13. Moccia, V.; D'Alessio, J. Burning behaviour of high-pressure $\mathrm{CH}_{4}-\mathrm{H}_{2}$-air mixture. Energies 2013, 6, 97-116.

14. Addabbo, R.; Bechtold, J.K.; Matalon, M. Wrinkling of spherically expanding flames. Proc. Combust. Inst. 2002, 29, 1527-1535.

15. Darrieus, G. Propagation d'un front de flame. La Tech Moderne 1938, 30, 18. (In French) 
16. Williams, F.A. Combustion Theory; The Benjamin/Cummings Publishing Company, Inc.: Menlo Park, CA, USA, 1985.

17. Kwon, O.C.; Rozenchan, G.; Law, C.K. Cellular instabilities and self-acceleration of outwardly propagating spherical flames. Proc. Combust. Inst. 2002, 29, 1775-1783.

18. Hu, E.; Huang, Z.; He, J.; Miao, H. Experimental and numerical study on laminar burning velocities and flames instabilities of hydrogen-air mixtures at elevated pressures and temperatures. Int. J. Hydrog. Energy 2009, 34, 8741-8755.

19. Wu, F.; Jomaas, G.; Law, C.K. An experimental investigation on self-acceleration of cellular spherical flames. Proc. Combust. Inst. 2013, 34, 937-945.

20. Tse, S.D.; Zhu, D.L.; Law, C.K. Morphology and burning rates of expanding spherical flames in $\mathrm{H}_{2} / \mathrm{O}_{2}$ /inert mixtures up to 60 atmosphere. Proc. Combust. Inst. 2000, 28, 1793-1800.

21. Law, C.K.; Jomaas, G.; Bechtold, J.K. Cellular instabilities of expanding hydrogen/propane spherical flames at elevated pressures: Theory and experiment. Proc. Combust. Inst. 2005, 30, 159-167.

22. Tang, C.; Huang, Z.; Wang, J.; Zheng J. Effects of hydrogen addition on cellular instabilities of the spherically expanding propane flames. Int. J. Hydrog. Energy 2009, 34, 2483-2487.

23. Zheng, S.; Zhang, X.; Xu, J.; Biao, J. Effects of initial pressure and hydrogen concentration on laminar combustion characteristics of diluted natural gas-hydrogen-air mixture. Int. J. Hydrog. Energy 2012, 37, 12852-12859.

24. Pelce, P.; Clavin, P. Influence of hydrodynamics and diffusion upon the stability limits of laminar premixed flames. J. Fluid Mech. 1982, 124, 219-237.

25. Patanaik, G.; Kailasanath, K.; Oran, E.S. Effect of gravity on flame instabilities in premixed gases. Am. Inst. Aeronaut. Astronaut. J. 1991, 29, 2141-2148.

26. Sinibaldi, J.O.; Mueller, C.J.; Tulkki, A.E.; Driscoll, J.F. Suppression of flame wrinkling by Buoyancy: The baroclinic stabilization mechanism. Am. Inst. Aeronaut. Astronaut. J. 1998, 36, 1432-1438.

27. Kadowaki, S. The body-force effect on the cell formation of premixed flames. Combust. Flame 2001, 124, 409-421.

28. Pan, K.L. Transformation of premixed flame behavior with hydrodynamic and body-force instabilities. Phys. Fluids 2008, 20, 044106.

29. Morley, C. Gaseq: A chemical equilibrium program for Windows. Available online: http://www.c.morley.dsl.pipex.com/ (accessed on 1 June 2014).

30. Bradley, D.; Lawes, M.; Liu, K.; Verhelst, S.; Woolley, R. Laminar burning velocities of lean hydrogen-air mixtures at pressures up to 1.0 MPa. Combust. Flame 2007, 149, 162-172.

31. Groff, E.G. The cellular nature of confined spherical propane-air flames. Combust. Flame 1982, $48,51-62$.

32. Bradley, D.; Harper, C.M. The development of instabilities in laminar explosion flames. Combust. Flame 1994, 99, 562-572.

33. Law, C.K.; Jomaas, G.; Bechtold, J.K. On transition to cellularity in expanding spherical flames. J. Fluid Mech. 2007, 583, 1-26.

(C) 2014 by the authors; licensee MDPI, Basel, Switzerland. This article is an open access article distributed under the terms and conditions of the Creative Commons Attribution license (http://creativecommons.org/licenses/by/3.0/). 